\title{
Evaluación del plan de administración ambiental para la granja avícola dos hermanos
}

\section{Evaluation of the plan of environmental administration for the farm poultry two brothers}

\author{
María Belén Parra Berrones \\ Luis Eduardo Hidalgo Almeida \\ Nelly Ivonne Guananga Díaz \\ Diego Iván Cajamarca Carrazco \\ Escuela Superior Politécnica de Chimborazo, Ecuador
}

Autor para correspondencia: diego.cajamarca@espoch.edu.ec

Fecha de recepción: 23 de agosto de 2018 - Fecha de aceptación: 01 octubre de 2018

Resumen: La presente investigación tiene como objetivo evaluar el Plan de Administración Ambiental de la Granja Avícola "Dos Hermanos" situada en la provincia de Chimborazo, cantón Chambo, por tratarse de un estudio de diagnóstico de contaminación e impacto ambiental, no se consideran tratamientos ni repeticiones, y no se ajusta a un Diseño Experimental, sino que responde al análisis de muestras compuestas de las aguas residuales tomadas en las instalaciones de la granja avícola cada 15 días para enviarlas al laboratorio. Los resultados indican el mejoramiento en las condiciones ambientales tanto de la granja como del medio circundante, puesto que los impactos negativos fueron controlados en un alto porcentaje. En las listas de chequeo los problemas ambientales más significativos fueron los tratamientos de los residuos sólidos determinando que un $52.08 \%$, de las actividades tienen no cumplimiento de los parámetros. Los puntos críticos que fueron identificados en el trabajo que le precede fueron mitigados sin embargo existen ciertos aspectos sobre todo en el área de bodegas, tanto de huevos como de alimento balanceado pese a que tienen empresas que controlan vectores no se pueden erradicar totalmente. El análisis general, en promedio, los impactos registraron una valoración de - 0.3 puntos de -3 posibles (para impactos de naturaleza negativa). Por lo expuesto se recomienda verificar que los contenedores de residuos estén cerrados, limpios y en orden y depositar los desechos en la planta procesadora y en tanques asignados.

Palabras Claves: condición ambiental; avícola; administración; contaminación; impacto

\begin{abstract}
The purpose of the current research is to evaluate an Environmental Administration Plan for "Dos Hermanos" Poultry Farm located in Chimborazo Province, Chambo County. Since it is an environmental impact and pollution diagnose study, it does not consider nor treatments or repetitions and is not under an experimental design; but it has to do with the analysis of sewage compound samples taken from the poultry farm facilities each 15 days to be sent to the laboratory. The results reflect improvement under environmental conditions both, in the farm and the surrounding environment, since the negative impacts were controlled in a high percentage. In the checklists, the most significant environmental problems were the solid waste treatments which determined that $52.08 \%$ of the activities do not accomplish the parameters. The critical points were
\end{abstract}


identified and mitigated in the previous work, however there are certain aspects especially in the warehouses area, both in eggs and in balanced food, but despite of having companies in charge of controlling vectors it is not possible to eradicate such aspects totally. The average general analysis of the impacts registers a value of -0.3 points of -3 possible (for negative nature impacts). Therefore, it is recommended to verify that the waste containers are closed, cleaned and ordered to place the waste from the processing plant in assigned containers.

Key Words: environmental conditions; poultry; administration; contamination; impact

\section{Introducción}

La industria avícola se ha convertido en un pilar fundamental para el sector agropecuario ecuatoriano, donde los sistemas modernos de crianza de aves son planificados bajo estrictos programas de sanidad, prevención y cuidado. En los procesos que se realizan en la industria avícola desde la crianza, donde además de ubicar a las aves en reducidas áreas hasta finalmente su beneficio se producen diferentes tipos de residuos como excretas, plumas, cascara de huevo respectivamente en volúmenes muy altos que si no son tratados y dispuestos adecuadamente se pueden transformar en serios problemas ambientales por lo que deben ser tratados de forma racional para hacer de esta actividad una práctica sostenible, que permita avanzar hacia una producción competitiva con un uso eficaz de los recursos manteniendo la armonía con el ambiente (Cañadas, 2016).

La evaluación de un plan de administración ambiental en el que se evalúa el grado de cumplimiento de las medidas de remediación, considerando las actividades que se realizan en las granjas avícolas permitirá determinar los principales focos de contaminación ambiental proponiendo nuevas soluciones viables para prevenir, mitigar y compensar los impactos negativos ocasionados optimizando de esta manera los recursos generados, obteniendo un mayor ingreso por el negocio de las explotaciones avícolas. La granja avícola Dos Hermanos es consciente de los procesos productivos que realiza y de su mejora ambiental de aquí la importancia de evaluar y restaurar el PMA realizado, con la finalidad de minimizar y corregir los actuales impactos ambientales negativos, cumpliendo de esta manera con las nuevas exigencias que la explotación requiere conteniendo en primera instancia un diagnóstico de los procesos productivos que ejecuta la granja y el impacto infringido sobre el área de influencia directa e indirecta, (Chavarrea, 2014). En el cual se incluye identificación y valoración de los principales focos de contaminación, así como las recomendaciones para el futuro control, seguimiento y mejoramiento de dichos impactos. Al manejar estas acciones no solo evitamos problemas dentro de la granja sino también fuera de está ayudando al entorno ambiental, mejorando de esta manera la relación con sus proveedores, ya que estos serán beneficiados con un producto de excelente calidad. De acuerdo con lo expuesto anteriormente se planteó: Evaluar el Plan de Administración Ambiental de la Granja Avícola "Dos Hermanos” del Cantón Chambo. 


\section{Materiales y métodos}

\section{Localización y duración del experimento}

La presente investigación se llevó acabo en las instalaciones de la granja avícola "Dos Hermanos" ubicada en el barrio el Batan del cantón Chambo, perteneciente a la provincia de Chimborazo, el estudio tuvo una duración de 65 días

\section{Unidades experimentales}

Las unidades experimentales que se consideraron para la presente investigación estuvieron conformadas por las muestras de aguas residuales provenientes de los procesos de producción de la granja avícola "Dos Hermanos".

\section{Tratamientos y diseño experimental}

Por tratarse de un estudio de diagnóstico de contaminación e impacto ambiental, no se consideraron ni tratamientos ni repeticiones en tal virtud no se ajusta a un Diseño Experimental, sino que responde a un análisis de las muestras compuestas de los residuos líquidos específicamente de aguas residuales que fueron tomadas en las instalaciones de la granja avícola "Dos Hermanos". El Estudio se basó en un diagnostico técnico mediante la matriz modificada de Leopald.

\section{Mediciones experimentales}

$\mathrm{PH}$

Sólidos Sedimentables.

Demanda Bioquímica de Oxigeno (DBO).

Demanda Química de Oxigeno (DQO).

Matrices de identificación de los impactos.

Matrices de valoración de los impactos.

Matrices de causa-efecto de los impactos.

\section{Análisis estadísticos y prueba de significación}

Por ser una investigación basada en la observación y el muestreo, los resultados experimentales fueron analizados mediante estadística descriptiva, en la cual se consideró: medidas de tendencia central (media, mediana, moda), medidas de dispersión (desviación estándar, error típico) y como prueba de significación se utilizó la prueba estadística T- student.

\section{Procedimiento experimental}

Se efectuaron visitas de observación, documentación fotográfica y entrevistas al personal que labora en la avícola, permitiendo recabar la información necesaria para conocer el estado actual de la granja y sus focos contaminantes con la finalidad de evaluar el grado de cumplimiento del Plan de manejo ambiental realizado anteriormente en sus instalaciones 
,formulando las nuevas medidas de remediación en base a sus exigencias, para mitigar, compensar y prevenir los efectos adversos, causados por los procesos productivos .

Se tomó 200 cc de los líquidos residuales, en vasos previamente esterilizados. Con las manos debidamente protegidas, para luego ser, identificados y transportados por medio de un termo al laboratorio SAQMIC para realizar los análisis respectivos de control de calidad.

La toma de muestras se ejecutó cada 15 días, en un periodo de dos meses (4 muestras) a la salida del galpón avícola.

Se efectuó una recopilación de datos para efectuar las matrices modificadas Leopald y de esa manera conseguir la calificación final de la industria Avícola "Dos hermanos".

\section{Resultados y discusión}

\section{Levantamiento de la línea base de la granja avícola "dos hermanos"}

\section{Ubicación y localización de la granja}

La granja avícola “Dos Hermanos”, está ubicada en el barrio, El Batán, perteneciente al cantón Chambo, provincia de Chimborazo, las coordenadas obtenidas a través del sistema de posicionamiento global (GPS) a las once horas fue de $1^{\circ} 25^{\prime} 0^{\prime \prime} \mathrm{S}$ y $78^{\circ} 30^{\prime} 0^{\prime \prime} \mathrm{W}$ en formato DMS (grados, minutos, segundos) o -1.41667 y -78.5 (en grados decimales)

\section{Condiciones ecológicas y edáficas de la granja}

La granja se encuentra localizada en una extensión de terreno fértil, rodeado de cercas naturales las cuales se encargan de mitigar los impactos ambientales generados por los diferentes procesos productivos que desarrolla la granja, son terrenos no aptos para el uso urbano, por su destinación a usos ganaderos, forestales, agrícolas, explotación de recursos naturales y control de procesos erosivos, así como zonas de protección forestal.

Esta zona pertenece a la formación de bosque húmedo Montano Bajo (b.h.MB.), según la clasificación ecológica de Holdridge, presenta variaciones micro-climáticas de acuerdo con los pisos altitudinales de las cordilleras, con precipitaciones entre 1000 y $2000 \mathrm{~mm}$.

\section{Presentación de la empresa}

La explotación avícola "Dos Hermanos" se encuentra ubicada en el barrio El Batan perteneciente al cantón Chambo, provincia de Chimborazo. La granja está constituida desde hace 12 años, actualmente cuenta con 16.000 aves, las mismas que una vez alcanzado las 18 a 19 semanas empiezan su primer ciclo de postura, la granja se dedica a la venta de huevos, y a la comercialización de pollonas (14 semanas), bajo pedido específicamente, la granja cuenta con su propia planta de balanceados y el suministro de alimento se lo realiza en base a los requerimientos de cada galpón. 
En cuanto al nivel organizacional de la empresa está a cargo del Ing. Zootecnista Daniel Paca, gerente propietario, quien ejecuta el manejo técnico de las aves y además cuanta con una persona que colabora en el proceso de producción que tiene un grado de tercer nivel en el área zootécnica y que asegura el manejo tecnificado de las aves. El área total de la granja es de 1 hectárea donde funciona 1 galpón de levante, 5 galpones de postura, bodega de almacenamiento, y la planta de balanceados.

\section{Actividad principal a la que se dedica}

La granja avícola "Dos Hermanos "se dedica principalmente a la producción de aves de postura, las mismas que alcanzado la edad de 18 a 19 semanas comienzan su primer ciclo de postura, tiene como propósito principal la venta de huevos y bajo pedido comercializa pollonas de 14 semanas a más de eso comercializa la gallinaza obtenida de cada uno de los galpones, esta actividad se la realiza cada seis meses.

\section{Política y organización de la empresa}

La empresa ha realizado una evaluación de los impactos ambientales que se producen en las distintas áreas de producción de aves de postura con la finalidad de disminuir los riesgos ambientales que esta actividad genera, tomando medidas correctivas para minimizar los impactos generados dentro de la granja y el área circundante. La empresa después de haber ejecutado un plan de administración ambiental pretende realizar la evaluación de este para verificar el grado de cumplimiento y mejorar las actividades que se realizan dentro de la granja sin alterar el ambiente originario de la comunidad

\section{Problemática del sector}

El cuidado del planeta es responsabilidad de la humanidad porque es primordial mantener un equilibrio entre la producción agropecuaria y la naturaleza. En los actuales momentos la producción avícola se ha integrado a la cadena productiva primaria del cantón Chambo, el cual presenta un clima diverso y agradable, ofreciendo condiciones favorables por su diversidad de pisos altimétricos y condiciones climáticas que son aptas para la producción pecuaria. Con respecto a la industria avícola son conocidas las causas que provocan los daños y alteraciones ambientales. Sin embargo, hoy en día existen las medidas afectivas para prevenir y mitigar los impactos negativos provocados por los procesos productivos de la producción avícola.

\section{Revisión ambiental inicial}

La granja se encuentra localizada en el barrio El Batán, perteneciente al cantón Chambo, está rodeada por explotaciones especialmente ganaderas, y pequeños núcleos familiares dedicados a la actividad de la agricultura, ganadería, explotación de recursos, así como la fabricación de ladrillos. Las acciones de remediación propuestas en el PMA con referencia a la ubicación de la granja han sido cumplidas en su totalidad, en estas se propuso la siembra de una mayor cantidad de especies vegetales para atrapar los malos olores La granja avícola dos hermanos está rodeada por una cortina vegetal constituida especialmente de eucaliptos, aguacates 
y manzanos que se encargan de purificar el aire atrapando los malos olores producto de las heces de las aves, evitando la contaminación a la atmosfera por la actividad pecuaria.

\section{Área de almacenamiento del balanceado}

La granja cuenta con su propia planta procesadora de alimento balanceado en la cual se elabora 2 toneladas de alimento al día, mismo que es distribuido a los 6 galpones de producción, las acciones de remediación propuestas en el PMA realizado, con respecto al área de almacenamiento no han sido cumplidas en su totalidad. Al no cumplirse con las acciones de remediación establecidas en el PMA que indica que, el área de ubicación del balanceado debería ser en silos para evitar la contaminación, desperdicio y perdida de la calidad de los nutrientes, así como también se deberá adecuar el área de bodega para el almacenamiento de huevos ya que al ser colocados en el interior del galpón los compradores tienen apertura al ingreso llevando consigo vectores infecciosos.

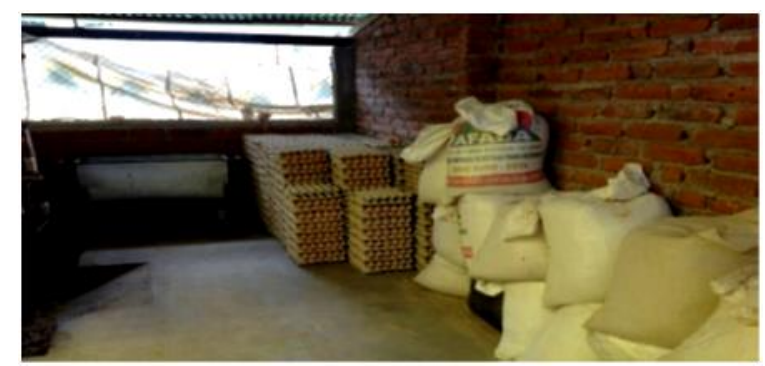

Fotografía 1. Área de almacenamiento de balanceado. Fuente: (Parra, 2018).

\section{Interior del galpón}

El área interna del galpón se encuentra constituida por jaulas, tolvas, niples y la fosa donde se albergan los desechos sólidos (huevos rotos, plumas, estiércol de las aves y desperdicio del alimento balanceado). Las acciones de remediación propuestas en el PMA realizado anteriormente en sus instalaciones han sido cumplidas en su totalidad, las fosas debajo de las jaulas poseen una altura aproximada de $50 \mathrm{~cm}$, impidiendo la acumulación de los residuos sólidos, evitando que estos entren en contacto directo con las jaulas de las ponedoras y las bandejas de recolección de huevos. Al haberse cumplido con las acciones de mitigación establecidas en el PMA se propone que se debería utilizar enzimas digestivas que ayuden a degradar más rápido el estiércol, estás deberían ser administradas en el alimento para disminuir los olores emanados por las aves ya que estos resultan perjudiciales para la salud de las mismas

\section{Almacenamiento de sacos}

El almacenamiento de sacos de las materias primas se lo realiza en una bodega localizada en la planta de balanceados, estos no están dispuestos de una forma ordenados por lo que atraen la presencia de ratas y ratones los cuales pueden trasportar enfermedades, además que son considerados como plagas en las explotaciones avícolas, sin embargo, cada dos meses los sacos son desalojados de la bodega por un comerciante quien se encarga de clasificarlos según su tamaño. 
Como se ha descrito en el PMA, que antecede a la presente investigación, se recomendó la creación de un espacio adecuado para el almacenamiento de los sacos, sin embargo no ha sido cumplido por lo tanto sería recomendable que se insista en la adecuación de un lugar específico, que disponga de áreas donde se puede colocar en forma ordenada los sacos, para evitar la proliferación sobre todo de ratas que se acercan a consumir el remanente de alimento que queda en cada uno de ellos y que al depositar sus heces u orina pueden provocar enfermedades

\section{Checklist para la identificación de los impactos ambientales en la granja "Dos hermanos"}

Los problemas fundamentales que están relacionados a la actividad de crianza de las aves, que pueden tener un impacto ambiental representativo así como también un impacto económico en el plantel son la sanidad animal y control de plagas, para la evaluación se estudió mediante encuestas cómo se maneja el control de plagas en el plantel avícola en lo cual se incluyó manejo, control de presencia de roedores, insectos y plagas, de acuerdo con esto se reportaron 60 observaciones a las preguntas propuestas, de las cuales el $43.33 \%$ de los parámetros estudiados cumplen con lo establecido (26 respuestas), el $40 \%$ no cumplen con lo establecido debiendo tener cuidado con el manejo (24 respuestas), el 16.67\% no aplica (10 respuestas), esto quiere decir que no tienen conocimiento de lo evaluado ya que no laboran por un periodo de tiempo considerable en la planta o no conocen sobre estos detalles. En el control de la sanidad de las aves se analizó parámetros de control de sanidad, manejo de antibióticos así como almacenamiento de los mismos, para tener un conocimiento total de los parámetros que afectan a la calidad, se reportaron 93 observaciones a las preguntas propuestas, para lo cual se determinó que el $67.74 \%$ fueron respuestas de cumplimiento de los parámetros de evaluación escogidos en el Check List, el $20.43 \%$ no cumplen con las respuestas y el $11.83 \%$ no aplica por desconocimiento de los parámetros

\section{Análisis físico - químico de las muestras de agua recolectadas a la entrada y salida de los galpones en la avícola "dos hermanos"}

\section{PH}

Al realizar el análisis estadístico del pH del agua que circula en la explotación avícola "Dos Hermanos" no se determinó diferencias estadísticas $(\mathrm{P}>0,05)$, estableciéndose que para los afluentes (entrada) se reportó medias de pH igual a 6.51 con un error estadístico de 0.004, un valor de mediana de 6.51 y moda de 6.51, mientras tanto que a la salida de la granja el pH del agua reporto 6.70 con un error aleatorio de 0.11 , una mediana de 6.70 , así como una moda de 6.52

Variaciones en $\mathrm{pH}$ pueden presentar efectos marcados sobre cada uno de los niveles de organización de la materia viva, desde el nivel celular hasta el de ecosistemas presentando altas variaciones en estas cadenas (Brañez, 2016).

\section{Conductividad Eléctrica}

Al monitorear el agua durante un periodo de 2 meses a la entrada y salida de la granja avícola "Dos Hermanos" se determinaron diferencias estadísticas significativas $(\mathrm{P}<0,05)$ al comparar los dos sitios de muestreo, los resultados obtenidos a la entrada reportaron promedios 
de $236.25 \mu$ Siemens $/ \mathrm{cm}$ con un error estadístico de \pm 0.75 , una mediana de $236.00 \mu$ Siemens $/ \mathrm{cm}$ y una moda de $235.00 \mu$ Siemens/cm mientras que a la salida se reportó una media de 270.75 $\mu$ Siemens/cm, una mediana de 271.00 y una moda $271.00 \mu$ Siemens/cm con un error estándar de 14,58, como se indica en el cuadro 1. Lo que es corroborado con lo que indica (Quiles, 2015), quien manifiesta que la determinación de la conductividad eléctrica evalúa la capacidad del agua para conducir la corriente eléctrica, es una medida indirecta de la cantidad de iones en solución (fundamentalmente cloruro, nitrato, sulfato, fosfato, sodio, magnesio).

Cuadro 1. Resultados químicos y físicos del agua tomada a la entrada y salida de los galpones en la granja avícola "dos hermanos"

\begin{tabular}{|c|c|c|c|c|c|c|c|c|c|c|}
\hline & pH & & Conduct & ibilidad & Solide & totales & DQO & & DBO & \\
\hline Estadísticos & Entrada & Salida & Entrada & Salida & Entrada & Salida & Entrada & Salida & Entrada & Salida \\
\hline Media & 6.51 & 6.70 & 236.3 & 270.8 & 249.3 & 344.8 & 2.85 & 52.3 & 1.15 & 30.6 \\
\hline Error típico & 0.00 & 0.11 & 0.75 & 14.58 & 0.75 & 27.28 & 0.05 & 3.903 & 0.03 & 0.83 \\
\hline Mediana & 6.51 & 6.70 & 236 & 271.00 & 249.0 & 345.00 & 2.8 & 52.50 & 1.15 & 30.65 \\
\hline Moda & 6.51 & 6.52 & 235 & 296.00 & 248.0 & 392.00 & 2.8 & 59 & 1.10 & 32.00 \\
\hline $\begin{array}{l}\text { Desviación } \\
\text { estándar }\end{array}$ & 0.01 & 0.21 & 1.5 & 29.16 & 1.50 & 54.56 & 0.1 & 7.805 & 0.06 & 1.65 \\
\hline $\begin{array}{l}\text { Varianza de la } \\
\text { muestra }\end{array}$ & 0.00 & 0.04 & 2.25 & 850.3 & 2.25 & 2976.92 & 0.01 & 60.92 & 0.00 & 2.72 \\
\hline Carosis & 1.50 & -5.90 & -3.90 & -5.99 & -3.90 & -6.00 & 4 & $\begin{array}{l}- \\
5.918\end{array}$ & -6.00 & -5.84 \\
\hline $\begin{array}{l}\text { Coeficiente de } \\
\text { asimetría }\end{array}$ & 0.00 & 0.02 & 0.37 & 0.00 & 0.37 & 0.00 & 2 & $\begin{array}{l}- \\
0.014\end{array}$ & 0.00 & -0.03 \\
\hline Rango & 0.02 & 0.38 & 3 & 51.00 & 3.00 & 95.00 & 0.2 & 14 & 0.10 & 3.00 \\
\hline Mínimo & 6.50 & 6.52 & 235 & 245.0 & 248.0 & 297.00 & 2.8 & 45 & 1.10 & 29.00 \\
\hline Máximo & 6.52 & 6.90 & 238 & 296.0 & 251.0 & 392.00 & 3 & 59 & 1.20 & 32.00 \\
\hline Suma & 26.04 & 26.81 & 945 & 1083. & 997.0 & 1379.0 & 11.4 & 209 & 4.60 & 122.30 \\
\hline t student & 0.06 & ns & 0.03 & $*$ & 0.01 & $*$ & 7.45 & ns & 0.000 & $* *$ \\
\hline
\end{tabular}

Fuente: Parra, (2018).

\section{Contenido de sólidos totales}

Para los sólidos totales se reportó diferencias estadísticas significativas estableciéndose un valor promedio de $249,25 \mathrm{mg} / \mathrm{l}$, una moda de $248.00 \mathrm{mg} / \mathrm{l}$ y un error estadístico de \pm 0.75 $\mathrm{mg} / \mathrm{l}$, con un valor de mediana de $249,00 \mathrm{mg} / \mathrm{l}$, mientras que a la salida de la explotación el contenido de solidos totales reportó una media de $344,75 \mathrm{mg} / \mathrm{l}$ con un error estadístico de \pm 27.28 $\mathrm{mg} / \mathrm{l}$, un valor de mediana de $345.000 \mathrm{mg} / \mathrm{l}$ con una moda de $392.0 \mathrm{mg} / \mathrm{l}$. Al respecto (Conesa, 2014), manifiesta que el termino solidos hace alusión a materia suspendida o disuelta en un medio acuoso. La determinación de sólidos disueltos totales mide específicamente el total de residuos sólidos filtrables (sales y residuos orgánicos), a través de una membrana con poros de $2.0 \mu \mathrm{m}$ (o más pequeños). La presencia de sólidos disueltos puede afectar adversamente la calidad de un cuerpo de agua. Se considera generalmente que, concentraciones de sólidos disueltos totales en el rango 50 a $600 \mathrm{mg} / \mathrm{litro}$, el agua tiene un sabor agradable; y que se 
deteriora progresivamente cuando la concentración sobrepasa los 1000 mg/litro con un límite superior de $1500 \mathrm{mg} / \mathrm{litro}$

\section{Demanda química de oxigeno}

El análisis estadístico no reporto diferencias significativas, el agua de entrada reportó una media de $2.85 \mathrm{mg} / \mathrm{l}$, con un error estándar igual a $\pm 0.05 \mathrm{mg} / \mathrm{l}$, un valor de mediana igual a 2.80 $\mathrm{mg} / \mathrm{l}$ y un valor de moda igual a $2.80 \mathrm{mg} / \mathrm{l}$; mientras tanto que, a la salida de la explotación avícola reportó un valor de DQO igual a $52.25 \mathrm{mg} / \mathrm{l}$, con un error estadístico igual a $\pm 3.90 \mathrm{mg} / \mathrm{l}$, con un valor de mediana igual a $52.50 \mathrm{mg} / \mathrm{l}$, y un valor de moda igual a $59.00 \mathrm{mg} / \mathrm{l}$. Los problemas generados por el aumento del DQO tienen su fundamento en lo que señala (Baker, 2014), quien indica que la demanda química de oxigeno es una medida de la cantidad de oxígeno disuelto consumido, bajo condiciones preestablecidas por la oxidación química de la materia orgánica biodegradable presente en el agua. Se usan diferentes oxidantes, como el dicromato potásico o el permanganato potásico. Este ensayo permite medir la cantidad de compuestos orgánicos, sales minerales oxidables (como los sulfuros), ya sean biodegradables o no.

\section{Demanda bioquímica de oxigeno}

Para la demanda bioquímica de oxigeno (DBO5) a la entrada de la granja "Dos Hermanos" se reportó un promedio de $1.15 \mathrm{mg} / 1$, con un error estadístico de $\pm 0.03 \mathrm{mg} / \mathrm{l}$, un valor de mediana igual a $1.10 \mathrm{mg} / \mathrm{l}$ y un valor de moda igual a $1.15 \mathrm{mg} / \mathrm{l}$; para el agua a la salida de la granja se reportó valores de $30.58 \mathrm{mg} / \mathrm{l}$, con un error estadístico igual a $\pm 0.83 \mathrm{mg} / \mathrm{l}$, un valor de mediana igual a $30.65 \mathrm{mg} / \mathrm{l}$ y un valor de moda igual a $32.00 \mathrm{mg} / \mathrm{l}$. De acuerdo con lo que reporta (Murillo, 2017), los contaminantes del agua en función a la calidad o las características del agua se clasifican en físicos, químicos y biológicos. Estas impurezas por el lado de las características químicas deben su origen a contaminantes orgánicos e inorgánicos. Los contaminantes orgánicos dan como resultado la disminución del oxígeno, producto de la degradación biológica de los compuestos y el aumento de la demanda bioquímica de oxígeno

Matriz para la determinación de la valoración global de los impactos

Para la determinación de la valoración global se aplicó la ecuación descrita a continuación, la cual permite, considerando las ponderaciones de cada componente, integrar las respuestas obtenidas de cada componente en un solo valor que caracteriza, de manera global, a cada impacto. La ecuación de valoración global de los impactos que se utilizó en l evaluación fue la siguiente:

$$
V_{-} g=W_{-} \mathbf{i} * V_{-} \mathbf{i}+\mathbf{W}_{-} \mathbf{E}^{*} \mathbf{V}_{-} \mathbf{E}+\mathbf{W}_{-} \mathbf{D}^{*} \mathbf{V}_{-} \mathbf{D}
$$




\begin{tabular}{cc}
\hline PONDERACIÓN & VALOR \\
\hline Peso del Factor Intensidad, $W_{i}$ & 0.40 \\
Peso del Factor Extensión, $W_{E}$ & 0.40 \\
Peso del Factor Duración, $W_{D}$ & 0.20 \\
\hline
\end{tabular}

Cuadro 2. Ponderación de los factores de los impactos para la determinación de la valoración global Fuente: Parra, (2018).

Para establecer la valoración global del impacto, posteriormente al cálculo del valor global, se procedió a integrar la naturaleza del impacto a la valoración obtenida, para lo cual se utilizó la siguiente regla descrita en el método de referencia:

Se multiplico por +1 a los valores globales de los impactos positivos Se multiplico por -1 a los valores globales de los impactos negativos

En el análisis general se puede apreciar que, en promedio, los impactos registraron una valoración igual a -0.3 puntos de -3 posibles (para impactos de naturaleza negativa), lo cual significa que, a pesar de que se genere alteraciones al medio por el accionar del plantel pecuario (en vista a que el signo del impacto es negativo), no se genera la degradación permanente del entorno y el mismo puede tolerar los impactos generados y compensarlos de manera natural.

\section{Conclusiones y recomendaciones}

La evaluación del Plan de Administración Ambiental de la Granja Avícola "Dos Hermanos", determinó un mejoramiento en las condiciones ambientales tanto de la granja como del medio circundante, puesto que los impactos negativos fueron controlados en un alto porcentaje.

En las listas de chequeo los problemas ambientales más significativos fueron los tratamientos de los residuos sólidos determinando que un $52.08 \%$, de las actividades tienen un no cumplimiento de los parámetros.

La Revisión Ambiental Inicial de la granja Dos Hermanos determino que los puntos críticos que fueron identificados en el trabajo que le precede fueron mitigados sin embargo existen ciertos aspectos que no han sido mitigados y que se pone de manifiesto sobre todo en el área de bodegas, tanto de huevos como de alimento balanceado pese a que tienen empresas que controlan vectores no se pueden erradicar totalmente.

En el análisis general se puede apreciar que, en promedio, los impactos registraron una valoración igual a -0.3 puntos de -3 posibles (para impactos de naturaleza negativa), lo cual significa que, a pesar de que se genere alteraciones al medio por el accionar del plantel pecuario (en vista a que el signo del impacto es negativo), no se genera la degradación permanente del entorno y el mismo puede tolerar los impactos generados y compensarlos de manera natural. 
Las acciones de remediación de la granja se han centrado en el control de los residuos sólidos como son la gallinaza que todavía mantiene un problema por los olores al ambiente, es necesario verificar que los contenedores de residuos estén cerrados, limpios y en orden. Depositar los desechos en la planta procesadora y en tanques asignados.

De acuerdo a las conclusiones expuestas se determinaron las siguientes recomendaciones

Una vez analizados con mayor profundidad los componentes del ecosistema que conforma la granja avícola Dos Hermanos es recomendable que se concientice a las personas que laboran en la empresa de la necesidad de mantener un control más estricto de la deposición de residuos sólidos que provocan efectos negativos sobre las personas y los animales que forman parte de la granja

Es necesario realizar un control más estricto de vectores tanto de moscas y ratas para evitar la contaminación cruzada es decir que lleven en sus extremidades bacterias que afectan directamente a los usuarios y fauna de la granja.

Es necesario establecer medidas de bioseguridad que contemplen actividades muy sencillas como son la señalética apropiada tanto de las áreas como de los productos que se almacenan en cada una de ellas.

\section{Bibliografía}

Anon, A. (2014). La gallinaza. ¿Un problema un curso económico? Madrid, España: Revista selecciones avícolas.

Arboleda, J. (2018, Julio 12). Manual de Evaluación de impacto ambiental. From http://www.Manual_EIA.com.

Arellano, A. (2015). Tratamiento de Aguas Residuales. (2a ed. ed.). (2.ed, Ed.) Riobamba, Ecuador.: Edit. ESPOCH.

Armendáriz, J. (2008, Diciembre 17). Tratamiento de residuos orgánicos en granjas avícolas. From https://www.aguasresiduales.com.

Baker, L. (2014, Marzo 22). Clasificación de la contaminación ambiental. . From http://www.produccion_limpia.com

Belinj. (2015, Agosto 10). Consideraciones para reutilizar la cama en galpones avícolas. From http://www.consulssac.com.

Bonnet, T. (2013, Abril 29). Subproductos de la actividad avícola. From http://www.agrobit.com.ar.Info.

Brañez, R. (2016). "Manual de Derecho Ambiental Mexicano". México DF, México: Fondo de Cultura Económica.

Calpa, J. (2017). Formulación del PMA para la planta de acopio de Alimentos Del Valle Alival S.A. Universidad Tecnológica De Pereira, Facultad De Ciencias Ambientales. Pasto Nariño: San Juan De Pasto.

Canziani, P. Y. (2007). Cambio climático y desarrollo limpio en Argentina. I congreso Argentino de Nutrición Animal (pp. 63-67.). Buenos Aires, Argentina: Caena. 
Cañadas, L. (2016, Noviembre 15). Conceptos generales sobre contaminación ambiental. . From http://www.cuencarural.com/.2016.

Carrizo, J. (2015). Alimentación de la pollita y la ponedora comercial: programas prácticos. Jornadas profesionales de avicultura de puesta (pp. 3 - 16.). Barcelona - España: Real Escuela de Avicultura.

Ceuppens, A. (2014, Agosto 29). Medio Físico o Medio Natural. From http://www.ifc.org/ifcex/enviro.com.

Chavarrea, M. (2014, Diciembre 14). Estudios de Medio Socioeconómico y su influencia en la producción avícola. . From http://www.envoronmentalguidelines,com.

Ciriazy, W. (2007). Conservación de los recursos (2a ed. ed.). México DF, México: Fondo de Cultura Económica.

Coma, J. y. (2014). Producción ganadera y contaminación ambiental. . Barcelona, 22 y 23 de noviembre de 2004. Xx curso de especialización fedna.

Conesa, F. (2014). Auditorias medioambientales. Guía Metodológica. . (2. Ed., Ed.) Barcelona, España.: Edit. Mundi Prensa.

Enzensberger, H. (2016). Contribución a la crítica de la ecología política. (2a.ed., Ed.) Puebla, México. : edit. Georgescu-Roegen.

FAO. (2016, Enero 12). Normas para el control de la calidad del huevo. Quito, Ecuador: Organización de las Naciones Unidas para la Agricultura y la Alimentación.

Fernández, M. (2016, Agosto 05). Contaminación por fósforo procedente de la fertilización orgánica de suelos agrícolas. From http://www.ibader.org.

Flores, J. (2014, Julio 15). Proceso metodológico evaluación de impacto ambiental. From http:///www.fao.org/es/agricultura/bpa.

Gómez, O. (2014.). Evaluación del Impacto Ambiental. . (1. Ed., Ed.) Madrid,, España. : Edit. Agrícola Española S.A.

Ibarrola, J. (2016, Marzo 03). La contaminación de la atmosfera. From http://wwwbibliotecadigital.ilce.edu.mx.

Ivarez, R. (2015). Efectos que causa la producción avícola. Desarrollo sostenible y Medio ambiente. Lima - Perú: Modulo V.

Jácome. (2005). Alimentación de la pollita y la ponedora comercial: programas prácticos. Jornadas profesionales de avicultura de puesta (pp. 34 -35). Barcelona - España: Real Escuela de Avicultura.

Llorente, J. (2016, Marzo 22). Contaminación de los suelos como mitigarla. . From https://www.3tres3.com.

Lozada, A. (2016, Diciembre 19). Guía de Buenas Prácticas Ambientales para el Sector Avícola. . From http://www.caftadrenvironment.org.

Martin, R. (2012). Tecnología y métodos para la producción de abonos orgánicos a partir de camas avícolas. Memorias del II Taller Internacional de Agricultura Sostenible en condiciones de Montaña, 23 -34.

Mertens, L. (2006). Formación en sistemas de calidad. Experiencias industria de alimentos. Chihuahua, México., México: Edit. Alpina.

Moguel, Y. y. (2005). Contenido de algunos macro y micro minerales en las deyecciones avícolas en Yucatán. Yucatan: Tec Pec.

Morales, H. (2003.). Utilización de la gallinaza de aves reproductoras en la engorda intensiva de toretes Holstein. . Buenos Aires: Ciencias Agropecuarias. . 
Murillo, T. (2017). Manejo de Residuos en la Industria Avícola. Manejo de Residuos en la Industria Avícola. (pp. 34 -35). San José de Costa Rica: Edit. Congreso Nacional Agronómico.

Orjuela, Y. (2018). Propuesta Del Plan De Manejo Ambiental Para La Avícola Acapulco Ubicada En El Municipio San Antonio Del Tequendama Departamento De CundinamarcaColombia. Bogotá: Universidad Militar Nueva Granada.

Ortiz, A. (2014). Evaluación de los desechos de la industria cafetera y azucarera como camas avícolas en Guantánamo y su aprovechamiento en la alimentación de ovinos. La Habana, Cuba.: Tesis de Dr. Cien. Vet. Instituto de Ciencia Animal.

Pérez, J. y. (2017, Febrero 12). Análisis de Sostenibilidad de la Industria Avícola en Guatemala. From https://www.incae.edu.

Quiles, A. (2015). La producción avícola y su efecto sobre el ambiente. Murcia. España: Departamento de Producción Animal, Facultad de Veterinaria.

Roberts, E. (2006.). Manual de control de la calidad del aire. Madrid, España.: Edit. Mc GrawHill.

Rodríguez, J. (2009). Utilización de nitrógeno no proteico. En: Investigaciones básicas para la utilización de las excretas de aves en la alimentación de los rumiantes: (1. Ed., Ed.) La Habana, Cuba.

Rostagno, H. (2015). Impacto de la nutrición de pollos de engorde sobre el medio ambiente. Memorias XVIII Congreso Latinoamericano de la Avicultura. Congreso Latinoamericano de la Avicultura. (p. 431). Bolivia, Congreso Latinoamericano de la Avicultura.

Takai, H. (2016, Enero 19). La matriz de Leopold, como interpretarla. . From http://wwwapi.eoi.es.com.

Tulsmag. (2017, Marzo 25). Normas de Legislación ambiental. From http://www.ambiente.gob.ec/wp-content/uploads/downloads/2015/06/Texto-Unificadode-Legislacion-Secundaria-del-Ministerio-del-Ambiente.pdf

Valdivié, M. \&. (2014). Evaluación de desechos de la industria cafetalera y azucarera como camas avícolas en Guantánamo y su aprovechamiento en la alimentación de ovinos. Guantanamo: Ovipac.

Vargas, A. (2013). Residuos industriales líquidos: Conceptos básicos y formas de tratamiento. Chile: Tesis de grado. Facultad de Ciencias Físicas y Matemáticas.

Whitehead, D. (2016, Agosto 12). La administración avícola, para un ambiente sano. From http://www.siame.gov.com

Williams, C. (2013). Gestión de residuos de aves en los países en desarrollo. North Carolina State. Estados Unidos de América: University. Department of Poultry Science. Raleigh, NC.

Zublena, J. (2014). Excess soil levels of copper, zinc, and phosphorus due to poultry manure applications. Proceedings 21st Annual Carolina Poultry Nutrition Conference. .P.R. Ferket. Ed. Caroli. Charlotte, North Carolina. 